\title{
Avaliação da qualidade do sono em estudantes de Medicina
}

\author{
Assessing quality of sleep in medical students
}

\author{
PALAVRAS-CHAVE \\ - Distúrbios do sono por sonolência \\ excessiva \\ - Educação médica \\ - Estudantes de medicina \\ - Sono \\ - Transtornos do sono
}

\author{
KEY WORDS \\ - Disorders of excessive somnolence \\ - Education, medical \\ - Students, medical \\ - Sleep \\ - Sleep disorders
}

\begin{abstract}
R E S U M O
Os estudantes de Medicina compõem um grupo susceptível aos transtornos do sono, em razão da carga curricular em horário integral, das atividades extracurriculares e da forte pressão e estresse, com exigência de alto rendimento e tempo demandado em estudos. O objetivo deste trabalho foi avaliar os hábitos do sono dos estudantes da graduação e residentes da Faculdade de Medicina da Universidade Federal de Goiás, a fim de identificar os principais fatores que afetam a qualidade do sono. Foram analisados 276 estudantes, sendo $234(84,7 \%)$ acadêmicos e 42 (18,2\%) residentes. Aplicou-se o questionário Índice de Qualidade do sono de Pittsburg (PSQI) e a escala de sonolência de Epworth. A análise dos questionários revelou associação estatisticamente significante entre sonolência e qualidade do sono (RR:1,108; IC 95\% 1,0047-1,2240). O trabalho demonstrou que estudantes com sonolência diurna excessiva apresentam pior qualidade de sono; e uma variação significativa da prevalência da sonolência diurna excessiva e da qualidade ruim do sono entre as amostras de primeiro ano de graduação e residentes.
\end{abstract}

\section{A B S T R A C T}

As a group, medical students are particularly susceptible to sleep disorders, due to their full-time course load, extracurricular activities, and heavy pressure and stress, with the demand for high yield and extensive study time. The aim of this study was to evaluate the sleeping habits of medical students and residents at the School of Medicine, Federal University in Goiás, in order to identify the principal factors that affect quality of sleep. The study analyzed a sample of 276 individuals, including 234 (84.7\%) undergraduate medical students and $42(18.2 \%)$ residents. The study used the questionnaire for the Pittsburgh Sleep Quality Index (PSQI) and the Epworth Sleepiness Scale (ESS). Analysis of the questionnaires showed a statistically significant association between sleepiness and quality of sleep (RR: $1.108 ; 95 \%$ CI $1.0047-1.2240$ ). The study showed that students with excessive daytime sleepiness present worse quality of sleep, and that there was a significant variation in the prevalence of excessive daytime sleepiness and poor quality of sleep when comparing first year undergraduates and residents. 


\section{INTRODUÇÃO}

Desde a Antiguidade, o processo do sono gera curiosidade nos homens. Hipócrates, por exemplo, já associava a insônia ao aborrecimento e à tristeza, enquanto Aristóteles acreditava que o sono era necessário para manter a percepção, que, se utilizada ininterruptamente, se esgotaria ${ }^{1}$.

Apesar do interesse que o sono e seus distúrbios despertaram ao longo da história da humanidade, somente na segunda metade do século 20 o sono passou a interessar não apenas aos filósofos e poetas, mas também aos cientistas. Reconhece-se, nos dias atuais, a importância do sono como agente restaurador e homeostático, com nítida influência sobre o estado vígil do indivíduo ${ }^{1}$.

O sono é uma função biológica fundamental na consolidação da memória, na visão binocular, na termorregulação, na conservação e restauração da energia ${ }^{2}$, e na restauração do metabolismo energético cerebral ${ }^{3}$. Devido a essas importantes funções, as perturbações do sono podem acarretar alterações significativas no funcionamento físico, ocupacional, cognitivo e social do indivíduo, além de comprometer substancialmente a qualidade de vida ${ }^{4-6}$.

Os transtornos do sono trazem consigo diversas repercussões para os seres humanos, ao provocarem perda da qualidade de vida, disfunção autonômica, diminuição do desempenho profissional ou acadêmica, aumento na incidência de transtornos psiquiátricos e diminuição da vigilância, com prejuízos na segurança pessoal e consequente aumento do número de acidentes. As pessoas que dormem mal tendem a ter mais morbidades, menor expectativa de vida e envelhecimento precoce ${ }^{7}$.

Na sociedade moderna, os distúrbios do sono, especialmente a insônia e a sonolência diurna excessiva, são queixas comuns na população em geral. Estima-se que a prevalência de insônia nas populações varie de 30 a $50 \%^{8}$. Já a prevalência de insônia crônica é de cerca de $10 \%{ }^{8}$.

O ciclo sono-vigília é um ritmo circadiano, apresentando, em condições naturais, sincronização com fatores ambientais e oscilando com um período de 24 horas. A alternância do dia-noite (claro-escuro), os horários escolares, os horários de trabalho, horários de lazer, as atividades familiares, todos são fatores exógenos que sincronizam o ciclo sono-vigília. Além dessa sincronização ambiental, o ciclo sono-vigília é gerado e regulado endogenamente por uma estrutura neural localizada no hipotálamo, o núcleo supraquiasmático, considerado o relógio biológico para os mamíferos ${ }^{9}$.

Em condições naturais, os seres humanos precisam de certa regularidade no seu padrão do ciclo sono-vigília, porém existem dois grupos de fatores que agem de forma antagônica no processo de sincronização do ciclo para os estudantes do curso de Me- dicina e residentes: o ciclo claro-escuro juntamente com os fatores endógenos, que tendem a sincronizar o ciclo sono-vigília em um ritmo regular e com período de 24 horas; e, em oposição, as demandas acadêmicas e hospitalares, que tendem a reduzir o sono e a sincronizar o ciclo sono-vigília com um padrão diferente do ciclo claro-escuro 9 .

Essa oposição entre os dois fatores pode ser considerada uma situação de conflito, em que os estudantes e residentes precisam decidir entre manter a regularidade do ciclo sono-vigília e satisfazer as necessidades de sono, ou responder aos esquemas e às obrigações acadêmicas e hospitalares.

Além das atividades curriculares em horário integral, é importante destacar que esses estudantes, em busca de uma boa qualificação profissional, complementam suas formações médicas com tarefas extracurriculares, como cursos de núcleo livre, ligas acadêmicas, plantões, estágios, iniciações científicas e monitorias. Assim, submetem-se a forte pressão e estresse pela exigência de alto rendimento e pelo tempo demandado em estudos.

Dessa forma, considerando que na população dos estudantes de Medicina e residentes observa-se padrão irregular do ciclo sono-vigília e alta prevalência dos transtornos do sono ${ }^{10-18}$, além de uma escassez de pesquisas locais sobre o assunto, o presente trabalho se propôs a avaliar os hábitos do sono de estudantes da graduação e residentes da Faculdade de Medicina da Universidade Federal de Goiás (UFG), possibilitando identificar os principais fatores que afetam a qualidade do sono da amostra a ser analisada.

\section{METODOLOGIA}

A amostra foi composta por um número (n) de 276 estudantes, escolhidos de forma aleatória entre os 660 alunos do primeiro ao sexto ano da graduação e os 60 residentes, todos matriculados na Faculdade de Medicina da Universidade Federal de Goiás. Desse total, 190 alunos do primeiro ao quarto ano, 44 internos (estudantes do quinto e do sexto ano) e 42 residentes, de ambos os sexos, com idade igual ou superior a 18 anos e que firmaram o Termo de Consentimento Livre e Esclarecido.

A coleta de dados foi realizada por meio da aplicação do Índice de Qualidade doSono de Pittsburgh (PSQI) e da Escala de Sonolência de Epworth (ESE), questionários autoaplicáveis, aos estudantes de Medicina e residentes.

Para avaliar a qualidade do sono da amostra, foi utilizado o PSQI, que apresenta sensibilidade de $89,6 \%$ e especificidade de $86,5 \%{ }^{19}$. Quando traduzido e validado para o português, o PSQI mantém sua alta sensibilidade ( $80 \%)$, no entanto uma especificidade ligeiramente menor, de $68,8 \%{ }^{20,21}$. 
Esse questionário contém dez questões. As de número um, dois, três e quatro possuem respostas do tipo abertas; e as questões de cinco a dez são objetivas. As questões cinco, nove e dez têm espaço para registro de comentários do entrevistado, caso haja necessidade.

As questões do PSQI formam sete componentes, que são analisados a partir de instruções para pontuação de cada um desses componentes, variando de zero a três pontos. A soma da pontuação máxima desse instrumento é de 21 pontos, sendo os escores superiores a cinco pontos indicativos de qualidade ruim no padrão de sono.

A avaliação específica dos componentes do PSQI ocorre da seguinte forma: o primeiro se refere à qualidade subjetiva do sono, ou seja, a percepção individual a respeito da qualidade do sono; o segundo demonstra a latência do sono, correspondente ao tempo necessário para iniciar o sono; o terceiro avalia a duração do sono, ou seja, quanto tempo permanece dormindo; o quarto indica a eficiência habitual do sono, obtido por meio da relação entre o número de horas dormidas e o número de horas em permanência no leito, não necessariamente dormindo; o quinto remete aos distúrbios do sono, ou seja, a presença de situações que comprometem as horas de sono; o sexto componente analisa o uso de medicação para dormir; o sétimo é inerente à sonolência diurna e aos distúrbios durante o dia, referindo-se às alterações na disposição e entusiasmo para a execução das atividades rotineiras.

Para a avaliação da sonolência diurna excessiva (SDE), utilizou-se a Escala de Sonolência de Epworth (ESE) publicada por W. Johns, já testada e validada para língua portuguesa ${ }^{22,23}$. Estudos clínicos com polissonografia, padrão-ouro para o diagnóstico dos distúrbios do sono, demonstraram que medidas na escala de Epworth acima de 10 estão associadas a distúrbios do sono ${ }^{10}$.

Esta escala vem acompanhada das instruções para pontuação das situações indagadas, tais como chance de cochilar sentado, lendo ou assistindo à televisão. A pontuação é indicada pelo estudante de acordo com as seguintes instruções: 0 corresponde a "não cochilaria nunca"; 1 corresponde a "pequena chance de cochilar"; 2 corresponde a "moderada chance de cochilar"; e 3 corresponde a "grande chance de cochilar". A pontuação indicada pelo estudante em todas as situações indagadas é somada e analisada. Resultados entre 0 e 10 pontos indicam ausência de sonolência; entre 10 e 16 pontos, sonolência leve; entre 16 e 20 pontos, sonolência moderada; e entre 20 e 24 pontos, sonolência severa.

Os dados coletados foram armazenados e analisados no programa Epi Info versão 3.2.2 e convertidos em planilhas do programa Microsoft Excel, para a confecção de gráficos e tabelas. Na análise dos dados quantitativos utilizaram-se medidas de tendência central, distribuição qui-quadrado e teste não paramétrico de Taylor. Os valores foram considerados estatisticamente significantes quando $\mathrm{p}=0,05$ / intervalo de confiança de $95 \%$.

\section{RESULTADOS}

O perfil sociodemográfico da amostra dos 276 estudantes se encontra na Tabela 1.

Tabela 1

Perfil Sócio-demográfico dos Estudantes e Residentes $(n=276)$

\begin{tabular}{|c|c|c|}
\hline Variável & $\mathrm{N}$ & $\%$ \\
\hline \multicolumn{3}{|l|}{ Gênero } \\
\hline Masculino & 151 & 54,7 \\
\hline Feminino & 118 & 42,8 \\
\hline Ignorado & 7 & 2,5 \\
\hline \multicolumn{3}{|l|}{ Idade } \\
\hline De 18 a 24 anos & 212 & 76,9 \\
\hline De 25 ou mais & 55 & 19,9 \\
\hline Ignorado & 9 & 3,2 \\
\hline \multicolumn{3}{|l|}{ Categoria } \\
\hline Acadêmico & 234 & 84,7 \\
\hline Residente & 42 & 15,3 \\
\hline \multicolumn{3}{|c|}{ Ano de graduação } \\
\hline $1 \mathrm{o}$ & 44 & 15,9 \\
\hline $2 \mathrm{o}$ & 48 & 17,4 \\
\hline 3 o & 48 & 17,4 \\
\hline 4 o & 50 & 18,1 \\
\hline Internos (5으 e 6응 & 44 & 15,9 \\
\hline Residentes & 42 & 15,2 \\
\hline
\end{tabular}

Por meio do questionário PSQI verificou-se que os estudantes demoravam, em média, 15,31 minutos para pegar no sono, com uma média de horário para acordar às 6 horas e 26 minutos da manhã. A duração média do sono na amostra foi de 6,13 h, e $87 \%(n=240)$ dos entrevistados apresentaram eficiência habitual do sono maior que $85 \%$. 
Observou-se que $56,5 \%(n=156)$ dos estudantes classificaram a qualidade do seu sono no período do último mês como boa e que $22,8 \%(n=63)$ a classificaram como ruim. A análise dos componentes do PSQI demonstrou que $14,9 \%(n=41)$ dessa amostra apresentavam qualidade ruim no padrão de sono, com diferentes prevalências dentre o ano de formação médica do estudante (Gráfico 1). Não foram observadas diferenças estatisticamente significantes na qualidade do sono entre os sexos ( $p=0,502)$ e entre os estudantes do primeiro ao sexto ano da graduação $(p=0,236)$. Contudo, essa diferença foi significativa quando comparado o padrão de sono dos residentes com o padrão do grupo de estudantes do primeiro ano de graduação $(p=0,050)$.

Entre os distúrbios do sono identificados na amostra pelo questionário PSQI inerentes ao mês anterior à entrevista, destaca-se que $32 \%(n=88)$ dos estudantes apresentaram sonhos ruins ou pesadelos menos de uma vez por semana; $24 \%(n=66)$ referiram problemas para dormir por sentirem muito calor; $22,1 \%(n=61)$ relataram acordar no meio da noite ou de manhã muito cedo uma ou duas vezes por semana. Além disso, 8,7\% $(n=24)$ dos participantes da amostra revelaram fazer uso de medicação para dormir.

Os entrevistados que fizeram comentários na questão de número cinco $(18,8 \%$; $\mathrm{n}=52)$ apresentaram outras razões que ocasionavam problemas para dormir além daquelas propostas pelo questionário PSQI (Tabela 2).

Na questão nove, 6,2\% $(n=17)$ dos estudantes comentaram sua indisposição ou falta de entusiasmo para realizar as atividades diárias, relacionando a queda da produção diária às seguintes causas: "cansaço, atividades extenuantes, plantões, poucas horas de sono, anemia, má qualidade do sono, medicamento, hipotireoidismo, preguiça, sono excessivo e sonolência diurna".

Na questão dez, 22,8\% (n = 63) dos entrevistados comentaram as situações em que mais cochilam (Tabela 3). Além disso, 6,2\% (n =17) caracterizaram a situação do cochilo como inevitável/indispensável ( $3 \% ; n=8)$, incômodo/problema $(1,8 \% ; n=5)$, descanso $(1 \% ; n=3)$ ou desnecessário-indiferente $(0,4 \% ; n=1)$.

A análise dos dados obtidos pela Escala de Sonolência de Epworth demonstrou que 44,7\% $(n=123)$ dos estudantes tinham dificuldades para ficar acordados em determinadas situações referidas pelo menos uma vez por semana; e que apenas $9,4 \%$ ( $n=$ 26) não apresentaram nenhuma indisposição no mês passado. Encontrou-se sonolência em 51,5\% (n=138) da amostra, sem diferença estatística entre as os anos de graduação e residência $(\mathrm{p}=$ $0,90)$, bem como entre os sexos ( $p=0,53)$.

A análise concomitante dos questionários PSQI e da ESE revelou que há associação estatisticamente significante entre a sonolência diurna excessiva e a qualidade do sono (risco relativo: 1,108; IC 95\% 1,0047 -1,2240), em que 66,7\% $(n=42)$ das pessoas que apresentaram qualidade ruim do sono também apresentaram SDE.

Gráfico 1

Qualidade do sono estratificada por ano de graduação

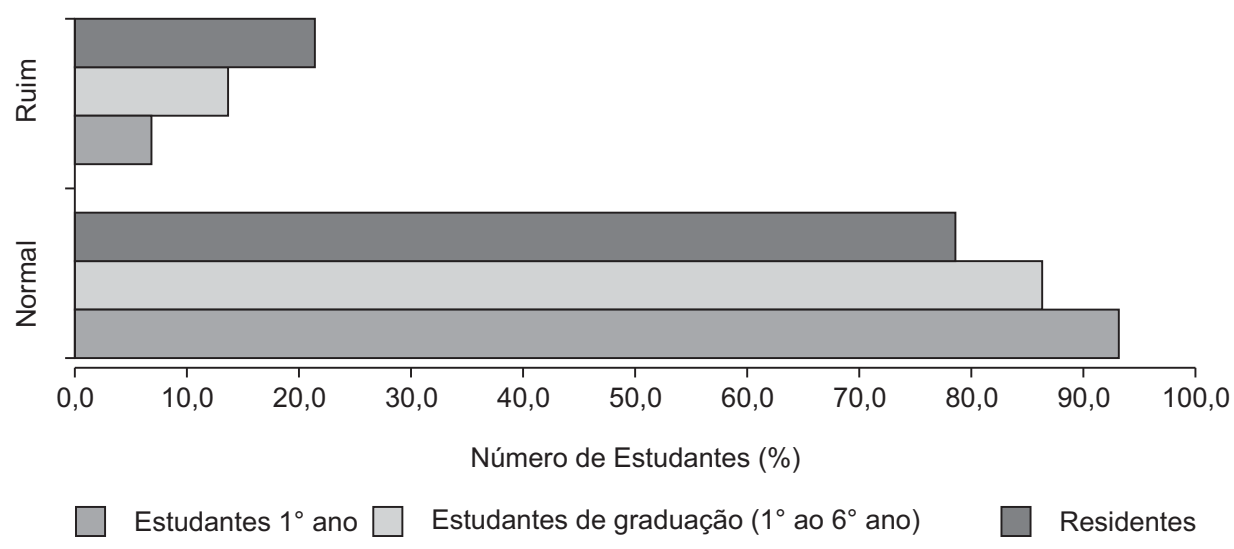

Dados para o gráfico 1 - Qualidade do sono estratificada por ano de graduação

\begin{tabular}{lccc} 
& $\begin{array}{c}\text { Estudantes } 1^{\circ} \text { ano } \\
\text { Normal }\end{array}$ & Estudantes de graduação $\left(1^{\circ}\right.$ ao $6^{\circ}$ ano $)$ & Residentes \\
Ruim & 93,2 & 86,3 & 78,6 \\
\hline
\end{tabular}


Tabela 2

Outras razões de problemas para dormir estratificadas por ano de graduação e sexo $(n=52)$

\begin{tabular}{|c|c|c|c|c|c|c|c|c|c|c|}
\hline & $1^{\mathrm{o}}$ & $2^{\mathrm{o}}$ & $3^{\mathrm{o}}$ & $4^{\mathrm{o}}$ & Internos & Residente & Masculino & Feminino & Sexo Ignorado & $f(\%)$ \\
\hline Preocupações & 3 & 9 & 1 & 3 & 1 & 0 & 7 & 10 & 0 & 32,7 \\
\hline Ansiedade & 1 & 2 & 4 & 0 & 1 & 0 & 2 & 6 & 0 & 15,4 \\
\hline Plantão & 0 & 0 & 0 & 0 & 1 & 4 & 4 & 1 & 0 & 9,7 \\
\hline Insônia & 1 & 0 & 1 & 1 & 1 & 0 & 2 & 2 & 0 & 7,7 \\
\hline Barulho & 0 & 1 & 2 & 0 & 1 & 0 & 3 & 1 & 0 & 7,7 \\
\hline Dores & 0 & 1 & 1 & 0 & 2 & 0 & 3 & 1 & 0 & 7,7 \\
\hline Disfunções orgânicas & 0 & 1 & 0 & 0 & 2 & 0 & 2 & 0 & 1 & 5,8 \\
\hline Nervosismo & 1 & 1 & 0 & 0 & 0 & 0 & 1 & 1 & 0 & 3,9 \\
\hline Sono diurno & 0 & 0 & 0 & 1 & 0 & 0 & 0 & 1 & 0 & 1,9 \\
\hline Álcool & 1 & 0 & 0 & 0 & 0 & 0 & 1 & 0 & 0 & 1,9 \\
\hline Outros & 0 & 1 & 1 & 1 & 0 & 0 & 1 & 2 & 0 & 5,8 \\
\hline Total & 7 & 16 & 10 & 6 & 9 & 4 & 26 & 25 & 1 & $52(100 \%)$ \\
\hline
\end{tabular}

Tabela 3

Situações em que cochila estratificadas por ano de graduação e sexo $(n=63)$

\begin{tabular}{|c|c|c|c|c|c|c|c|c|c|c|}
\hline & $1^{\mathrm{o}}$ & $2^{o}$ & $3^{o}$ & $4^{\mathrm{o}}$ & Interno & Residente & Masculino & Feminino & Ignorado & $f(\%)$ \\
\hline Após almoço & 2 & 4 & 3 & 3 & 4 & 1 & 12 & 3 & 2 & 27 \\
\hline Em aulas & 4 & 2 & 1 & 3 & 4 & 0 & 7 & 7 & 0 & 22,2 \\
\hline $\begin{array}{l}\text { Devido } \\
\text { ao cansaço }\end{array}$ & 1 & 2 & 3 & 0 & 2 & 2 & 7 & 3 & 0 & 15,9 \\
\hline $\begin{array}{l}\text { Devido } \\
\text { à sonolência }\end{array}$ & 1 & 1 & 1 & 2 & 0 & 2 & 5 & 2 & 0 & 11,1 \\
\hline $\begin{array}{l}\text { Quando } \\
\text { há tempo }\end{array}$ & 1 & 2 & 2 & 0 & 0 & 1 & 2 & 4 & 0 & 9,5 \\
\hline Estudando/ lendo & 0 & 1 & 0 & 1 & 2 & 2 & 2 & 4 & 0 & 9,5 \\
\hline Intervalos & 1 & 0 & 0 & 0 & 0 & 0 & 1 & 0 & 0 & 1,6 \\
\hline Vontade & 0 & 1 & 0 & 0 & 0 & 0 & 1 & 0 & 0 & 1,6 \\
\hline $\begin{array}{l}\text { Quando } \\
\text { acorda cedo }\end{array}$ & 0 & 0 & 1 & 0 & 0 & 0 & 0 & 1 & 0 & 1,6 \\
\hline Total & 10 & 13 & 11 & 9 & 12 & 8 & 37 & 24 & 2 & $63(100 \%)$ \\
\hline
\end{tabular}




\section{DISCUSSÃO}

A amostra de estudantes analisada apresentou uma média de horas dormidas por noite $(6,13 \mathrm{~h})$ inferior à média da população adulta em geral (de sete a nove horas) ${ }^{8}$. Contudo, deve-se lembrar que alguns indivíduos necessitam de menos horas de sono e outros requerem mais 8 .

A privação de sono pode explicar grande parte dos períodos de sonolência e dos distúrbios do sono apresentada pelos estudantes avaliados. Esse fato preocupa, já que há comprovação de que a privação do sono tem correlação significativa com a diminuição do desempenho acadêmico e profissional entre estudantes de Medicina e residentes ${ }^{11}$.

Ao se analisar a qualidade do sono de acordo com o período de formação na área médica, observou-se maior prevalência de pessoas com qualidade ruim do sono no grupo dos residentes $(21,4 \%)$, com diferença estatisticamente significante quando comparada ao padrão ruim de qualidade do sono do grupo de estudantes do primeiro ano de graduação (6,8\%). Esses resultados podem ser explicados pelos fatores de risco para insônia e distúrbios do sono apresentados pelos residentes, tais como idades superiores ${ }^{8}$ às dos alunos do primeiro ano, além de maior carga horária destinada ao processo de formação médica e às extenuantes atividades profissionais.

Além disso, na amostra de estudantes avaliada, evidenciou-se uma alta prevalência do uso de drogas com finalidades hipnóticas $(8,7 \%$; $=24)$ no mês anterior ao estudo. Essa taxa é superior às apresentadas por estudos semelhantes na população geral do Brasil $(6,9 \%)^{24}$ e dos Estados Unidos $(7,1 \%)^{25}$, em que esses autores concluíram que se tratava de uso excessivo de drogas hipnóticas sedativas.

A sonolência diurna excessiva foi encontrada em 51,5\% ( $\mathrm{n}=$ 138) da amostra estudada, uma prevalência superior à apresentada pela população em geral $(11,6 \% \text { a } 36 \%)^{12}$, mas inferior à encontrada em estudantes de Medicina chilenos $(93,2 \%)^{12}$.

Na amostra estudada, não foram observadas diferenças estatisticamente significativas das prevalências de SDE entre os sexos. Apesar de estudos populacionais demonstrarem maior ocorrência de SDE nos homens ${ }^{11}$, sabe-se que, quando os indivíduos são submetidos a um mesmo ambiente de trabalho, com exigências semelhantes, como ocorre entre os estudantes de Medicina, essa diferença de SDE diminui ${ }^{11}$.

\section{CONCLUSÕES}

Ao avaliar os hábitos do sono dos estudantes de Medicina em diferentes fases do curso, conclui-se que esse grupo dorme, em média, menor quantidade de horas, apresenta maior sonolência diurna e faz mais uso de drogas com finalidade hipnótica, quando comparado com a população adulta em geral. E os estudantes que apresentam pior qualidade de sono também são portadores de sonolência diurna excessiva.

Este estudo possibilitou, ainda, a identificação dos principais fatores do PSQI que afetam a qualidade do sono dos estudantes de Medicina (presença de sonhos ruins ou pesadelos; problemas para dormir decorrentes de condições climáticas, o fato de acordar no meio da noite ou de manhã muito cedo), além de fatores citados pela própria amostra: "preocupações, ansiedade, plantão, insônia, barulho, dores".

Dessa forma, recomenda-se aos docentes do curso de Medicina que planejem as aulas e as atividades extracurriculares com certa regularidade de horários, considerando, dentro do possível, os períodos de sonolência diurna e os de maior alerta/rendimento dos estudantes; e que promovam o desenvolvimento de programas preventivos que orientem os alunos sobre a importância da regularidade e das medidas de higiene do sono.

\section{REFERÊNCIAS}

1. Souza JC, Sousa NM. Sono, filosofia e mitologia: correlações clínico-filosóficas e mitológicas. 1ạ ed. Campo Grande: Editora da UCDB; 1998.

2. Reimão R. Sono: estudo abrangente. $2^{\underline{a}}$ ed. São Paulo: Atheneu; 1996.

3. Ferrara M, Gennaro L. How much sleep do we need? Sleep Med. 2001;5(2)155-79.

4. Bittencourt LRA, Silva RS, Santos RF, Pires MLN, Mello MT. Sonolência excessiva. Rev Bras Psiquiatr. 2005;27(Supl.1):16-21.

5. Souza JC, Souza NM, Arashiro ESH, Schaedler R. Sonolência diurna excessiva em pré-vestibulandos. J Bras Psiquiatr. 2007;56(3):184-7.

6. Muller MR, Guimarães SS. Impacto dos transtornos do sono sobre o funcionamento diário e a qualidade de vida. Estud Psicol. (Campinas). 2007;24(4):519-28.

7. Medeiros ALD, Lima PF, Almondes KM, Dias Junior AS, Rolim SAM, Araújo JF. Hábitos de sono e desempenho em estudantes de medicina. Revista Saúde do centro de ciências da saúde (UFRN) 2002;16(1)49-54.

8. Poyares D, Tufik S. I Consenso Brasileiro de Insônia: introdução. Hypnos: rev sono. 200315 out.; São Paulo: Sociedade Brasileira de Sono; 2003. p. 5. Oct:4-45.

9. Almondes KM, Araújo JF. Padrão do ciclo sono-vigília e sua relação com a ansiedade em estudantes universitários. Estud Psicol. 2003;8(1)37-43. 
10. Rodrigues RND, Viegas CAA, Silva AAAA, Tavares P. Daytime sleepiness and academic performance in medical students. Arq Neuropsiquiatr. 2002;60(1)6-11. Doi: 10.1590/S0004-282X2002000100002.

11. Danda GJN, Ferreira GR, Azenha M, Souza KFR, Bastos O. Padrão do ciclo sono-vigília e sonolência excessiva diurna em estudantes de medicina. J Bras Psiquiatr. 2005;54(2)102-6.

12. Santibañez I. Estudo de hábitos normais e patológicos de sono e vigília de estudantes de medicina: estudo de prevalência. J Bras Psiq. 1994;(43):33-7.

13. Mahendran R, Subramaniam M, Chan YH. Medical students' behaviour, attitudes and knowledge of sleep medicine. Singapore Med J. 2004;45(12)587.

14. Lima PF, Medeiros ALD, Rolim SAM, Dias Junior AS, Almondes KM, Araujo JF. Changes in medical students' sleep habits according to class starting time: a longitudinal study. Hypnos. 2004;1(1)10-9.

15. Medeiros ALD, Mendes DBF, Lima PF, Araújo JF. The relationships between sleep-wake cycle and academic performance in medical students. Biol Rhythm Res. 2003;32(2)263-70.

16. Lima PF, Medeiros ALD, Araujo JF. Sleep-wake pattern of medical students: early versus late class starting time. Braz J Med Biol Res. 2002;35(11)1373-7.

17. Loayza MPH, Ponte TS, Carvalho CG, Pedrotti MR, Nunes PV, Souza CM et al. Association between mental health screening by self-report questionnaire and insomnia in medical students. Arq Neuropsiquiatr. 2001;59(2-A):180-5.

Doi: 10.1590/S0004-282X2001000200005.

18. Gaspar S, Moreno C, Menna-Barreto L. Os plantões médicos, o sono e a ritmicidade biológica. Rev Assoc Med Bras. 1998;44(3)239-45. Doi: 10.1590/S0104-42301998000300013

19. Buysse DJ, Reynolds CF 3rd, Monk TH, Berman SR, Kupfer DJ. The Pittsburgh Sleep Quality Index: A new instrument for psychiatric practice and research. J Psychiatr Res. 1989;28(2)193-213.

20. Kawakami N, Takatsuka N, Shimizu H. Sleep disturbance and onset of type 2 diabetes. Diabetes Care. 2004;27(1)282-3.
21. Bertolazi AN, Fagondes SC, Perin C, Schonwald SV, John AB, Miozzo ICS, et al. Validation of the Pittsburgh Sleep Quality Index in the brazilian portuguese language. In: Sleep 2008. 22nd Annual meeting of the associated professional sleep societies; 2008; Baltimore. Sleep. Westchester: APSS; 2008. v. 31. p. a347.

22. Bertolazi AN, Barreto SSM, Drehmer M. Tradução, adaptação cultural e validação da Escala de Sonolência de Epworth. In: XXXIII Congresso Brasileiro de Pneumologia e Tisiologia; 2006; Fortaleza: Jornal Brasileiro de Pneumologia; 2006. v. 32 p. 217-402.

23. Bertolazi AN, Fagondes SC, Perin C, Schonwald SV, John AB, Miozzo ICS, et al. Validation of the Epworth Sleepiness Scale in the brazilian portuguese language. In: Sleep 2008 - 22nd Annual meeting of the associated professional sleep societies; 2008; Baltimore. Sleep. Westchester: APSS; 2008. v. 31. p. a347-a347.

24. Souza JC, Magna LA, Reimão R. Insomnia and hypnotic use in Campo Grande general population, Brazil. Arq Neuropsiquiatr. 2002;60(3B):702-7.

25. Nishino S, Mignot E. Drug treatment of patients with insomnia and excessive daytime sleepiness: pharmacological considerations. Clin Pharmacokinet. 1999;(37):305-30.

\section{CONTRIBUIÇÃO DOS AUTORES}

Todos os autores participaram na concepção e desenho deste estudo, coleta e interpretação dos dados e redação do deste texto.

\section{CONFLITO DE INTERESSES}

Declarou não haver.

\section{ENDEREÇO PARA CORRESPONDÊNCIA}
Hígor Chagas Cardoso
$1^{\text {a }}$ Avenida s/n
Campus Universitário - Goiânia
CEP.: 74605-020 GO
E-mail: medhigor@gmail.com 